\title{
Thermally Reversible Self-Assembly of Nanoparticles via Polymer Crystallization
}

\author{
Calum Kinnear, Sandor Balog, Barbara Rothen-Rutishauser, Alke Petri-Fink*
}

The directed self-assembly of gold nanoparticles through the crystallization of surface-grafted polyethylene oxide (PEO) in ethanol-water mixtures is described. This process is fully reversible and tunable through either the size of the core or the polymeric coating. Characterization by X-ray scattering and electron microscopy of the self-assembled structures reveals order at the nanoscale, typically not the case for thermoresponsive gold nanoparticles coated with lower or upper critical solution temperature polymers. A further novelty is the result of selective binding of calcium ions to the PEO in the fluid state: a reversible thermoresponsive transition become irreversible.

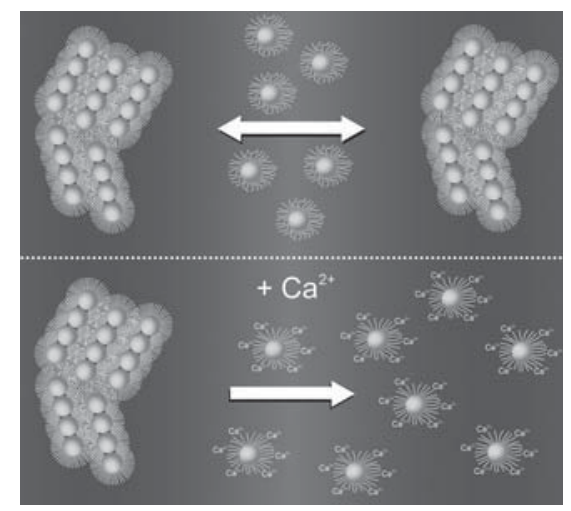

The exploitation of external stimuli to predictably control the dispersion state of nanoparticles (NPs) in suspension is an alluring prospect. ${ }^{[1]}$ Indeed, a variety of approaches have been developed to generate stimuli-responsive NPs whereby attractive forces between particles dominate the repulsive interactions leading to either dynamically arrested states, or self-assembled NP crystal structures. ${ }^{[2-4]}$ Gold NPs have garnered interest in this field due to their fascinating optical properties; spherical particles exhibit a deep ruby color due to their localized surface plasmon resonance (LSPR). ${ }^{[5]}$ The LSPR is extremely sensitive to the local dielectric environment; the adsorption of proteins or the close approach of another NP results in a measurable shift of the resonance. ${ }^{[6]}$ The directed self-assembly of gold NPs has led to the development of colorimetric sen-

Dr. C. Kinnear, Dr. S. Balog, Prof. B. Rothen-Rutishauser, Prof. A. Petri-Fink

Adolphe Merkle Institute, University of Fribourg, Chemin des Verdiers 4, Fribourg 1700, Switzerland

E-mail: alke.fink@unifr.ch

Dr. C. Kinnear, Prof. A. Petri-Fink

Department of Chemistry, University of Fribourg, Chemin du Musée 9, Fribourg 1700, Switzerland sors for the detection of heavy metals, ${ }^{[7]}$ polynucleotides, ${ }^{[8]}$ or antigens. ${ }^{[9]}$ Additionally, ordered arrays of NPs have been used in catalysis, ${ }^{[10]}$ solar energy harvesting, ${ }^{[11]}$ highdensity data storage, ${ }^{[12]}$ surface-enhanced Raman scattering, ${ }^{[13,14]}$ and metamaterials. ${ }^{[15]}$

In order to obtain a desired self-assembled structure in dilute systems, the correct strength and length scales of interactions between the constituent particles are needed. If the interaction acts over a short length scale relative to the size of the particles, or if it is too strong relative to thermal energy, then self-assembly may not occur due to crossing of the dynamical arrest phase boundary. ${ }^{[4]}$ In this phase, long-lived glasses and gels persist with low degrees of order and potential nonergodicity. Therefore, a judicious choice of system components is crucial to balance the strengths of attractive interactions, such as opposite electrostatic charges and van der Waals, with repulsive interactions such as steric effects or similar electrostatic charges. ${ }^{[2]}$ Indeed, gold NPs of different shapes have been self-assembled, with the appropriate choice of surface ligands and dissolved species in bulk, through the use of van der Waals interactions, ${ }^{[16]}$ depletion forces, ${ }^{[17,18]}$ hydrogen bonding, ${ }^{[19]}$ DNA hybridization, ${ }^{[20]}$ and long-range electrostatics. ${ }^{[21]}$ 
The concept of thermosensitive gold NP dispersions using surface-grafted polymeric ligands was first demonstrated by Zhu et al. ${ }^{[22]}$ with poly( $N$-isopropylacrylamide). This polymer presents a lower critical solution temperature (LCST) transition whereby above a certain temperature the chains collapse and become hydrophobic leading to aggregation, ultimately driven by entropy. ${ }^{[23]}$ The thermal behavior of grafted polyacrylamides has been investigated extensively, while only a few other polymers exhibiting an LCST have gathered much attention, such as poly $(N-$ vinylcaprolactam) ${ }^{[24]}$ or poly(vinyl methyl ether). ${ }^{[25]}$ Polymer-NP conjugates, termed thermoresponsive, typically aggregate into dynamically arrested aggregates that resemble gels or glasses, likely due to short ranged but strong van der Waals interactions, which are less dominant for self-assembled block copolymers. Contrary to the numerous systems known to exhibit LCST behavior in various solvents, upper critical solution temperature (UCST) polymers, which, driven by enthalpy, phase separate upon cooling below the transition temperature, are far less investigated. ${ }^{[26]}$

Polyethylene oxide (PEO) is a polymer that exhibits UCST behavior in water; however, it is practically inaccessible as the transition temperature lies above $100{ }^{\circ} \mathrm{C} .^{[27]}$ Nevertheless, the polymer has been widely investigated due to its simplicity, functionality, and amphilicity. When dissolved in ethanol-water mixtures, PEO exhibits unusual phase and thermal behavior with a UCST below the crystallization temperature. ${ }^{[28,29]}$ However, if the concentration of water is above a threshold, the ethylene oxide (EO) monomers are sufficiently solvated and do not crystallize.

Here, we report on an investigation into the use of PEO-grafted gold NPs as a reversible thermoresponsive system. The self-assembly of coated gold NPs was studied as a function of the core size and PEO molecular weight. Additionally, the self-assembled structure has been investigated with potentially different long-range order identified from lamellar-like domains to cubic close packing arrangements.

Three sizes of gold NPs $\left(\mathrm{Au}_{5}, \mathrm{Au}_{16}\right.$, and $\mathrm{Au}_{33}$ of 5,16 , and $33 \mathrm{~nm}$ core diameter) were functionalized with two different molecular weights of thiolated PEO (5 and $10 \mathrm{kDa}$ ) at a concentration of $10 \mathrm{PEG} \mathrm{nm}^{-2}$. Hereafter, the intermediate core size and polymer will be mainly discussed $\left(\mathrm{Au}_{16}\right.$ and $\left.5 \mathrm{kDa} \mathrm{PEO},[\mathrm{Au}]=1 \times 10^{-3} \mathrm{M}\right)$ unless otherwise specified. It was immediately evident that the grafted polymer was causing colloidal phase separation in absolute ethanol at room temperature as expected for a polymer exhibiting UCST behavior or crystallization (Figure 1a), and redispersion was a simple matter of running hot water over the vial. Given that the residual concentration of unreacted polymer must be less than $0.01 \times 10^{-6} \mathrm{M}$, equating to 0.3 molecules per $\mathrm{nm}^{2}$ of $\mathrm{NP}$ surface area (Supporting Information), the observed selfassembly must originate from grafted, rather than free, PEO.

A red-shift in the LSPR is an indication of gold NP aggregation or self-assembly, which was observed for both the $\mathrm{Au}_{16}$ and $\mathrm{Au}_{33}$ systems. Quenching the dispersion from above the melting transition, i.e., $40{ }^{\circ} \mathrm{C}$ to $15^{\circ} \mathrm{C}$, and delaying the measurement for 15 min resulted in a shift of 16-19 nm (Figure 1a). As expected, a larger and broader shift was found for the $\mathrm{Au}_{33}$ NPs with a similar sized PEO layer, while no shift was found for the $\mathrm{Au}_{5} \mathrm{NPs}$ (Figure S1, Supporting Information). ${ }^{[30]}$ The shift was reversible across multiple heating-cooling cycles, and a transition temperature of around $25{ }^{\circ} \mathrm{C}$ was identified from a cooling ramp (Figure 1b). Assuming the PEO coating presents a hard-core repulsion interaction potential, the minimum possible separation of two NPs is twice the thickness of the polymer layer. This layer is approximately $10 \mathrm{~nm}$ : the difference between the hydrodynamic radius from dynamic light scattering (DLS) and the NP radius from transmission electron microscopy (TEM). From the work of Tabor et al., ${ }^{[31]}$ we would expect an LSPR shift of less than $1 \mathrm{~nm}$ if the $\mathrm{Au}_{16}$ NPs were separated by $20 \mathrm{~nm}$ of PEO, not the 16-19 $\mathrm{nm}$ shift found. Despite this simplified view of ignoring multiparticle plasmon coupling and the competing van der Waals interaction with steric repulsion, ${ }^{[2]}$ the large shift in the LSPR is indicative of a much smaller interparticle spacing in the self-assembled structure than expected from a brush conformation PEO coating.

The self-assembly was probed by DLS and multiple heating-cooling cycles, with the hydrodynamic radius estimated via a cumulant analysis and the StokesEinstein relation. Starting around $18{ }^{\circ} \mathrm{C}$ for the $5 \mathrm{kDa}$ $\mathrm{PEO}$, and $24^{\circ} \mathrm{C}$ for the $10 \mathrm{kDa}$ PEO, an increase in the size was measured corresponding to the crystallization of the polymer and the self-assembly of NPs (Figure 1c). Upon heating, the assemblies rapidly break up at $38{ }^{\circ} \mathrm{C}$ due to the PEO adopting a brush conformation, now in a good solvent, with the steric repulsion overcoming the van der Waals attractions. A hysteresis was observed between the two phase transitions, mirrored in the heating-cooling cycles of the UV-vis-NIR spectra (Figure 1d).

The assembled particles were imaged by TEM, with large 3D micron-sized structures found (Figure 2 and Figure S2, Supporting Information). Ordered domains could be observed that resembled either lamellar or cubic forms. Indeed, the lamellar structure is reminiscent of the morphologies observed for spinodal decomposition or the microphase separation of a block copolymer, ${ }^{[32]}$ where in this case the inorganic gold core acts as the B block in an A-B-A copolymer. The separation distance between adjacent NPs was found to be less than the predicted thickness of a solvated PEO layer. This is not surprising as partial 

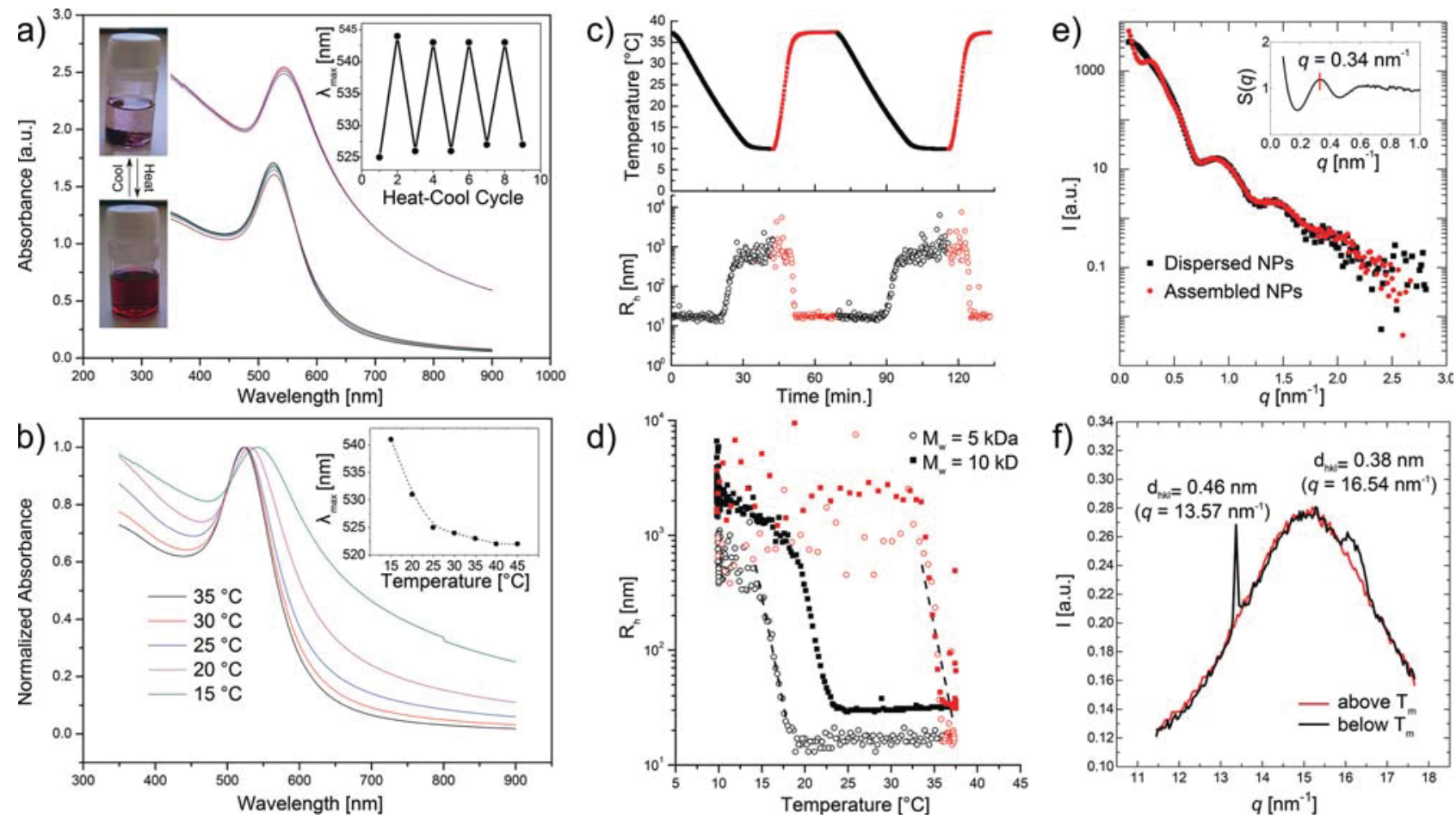

Figure 1. The self-assembly or dispersion of gold NPs upon cooling or heating. a) The LSPR of gold NPs was measured by UV-vis spectroscopy upon repeated heating-cooling cycles from 15 to $40^{\circ} \mathrm{C}$. Inset: Shifts in the LSPR maximum through heating-cooling cycles. b) The LSPR of gold nanoparticles at set temperatures during a cooling cycle. Inset: Position of the LSPR maximum with temperature. c) Temperature during the cooling (black) and heating (red) cycles (upper) and the corresponding hydrodynamic radius by DLS (lower). d) Self-assembly and dispersion hysteresis of NPs functionalized with two different molecular weights of PEO during cooling (black) and heating (red) cycles. e) SAXS curves of NPs either self-assembled in absolute ethanol, or well dispersed in a water-ethanol mixture. Inset: Structure factor for the self-assembled NPs with a maxima at $q=0.34 \mathrm{~nm}^{-1}$. f) WAXS curves of a 2 wt\% solution of PEO in ethanol either above or below the phase transition temperature.

compression, and interdigitation, of polymer brushes due to van der Waals attractions can occur especially when the size of the polymer brush is small with respect to the NP. ${ }^{[33,34]}$ Within the lamellar regions, the NPs are in near contact with a larger separation between the layers (Inset of Figure $2 \mathrm{~b}$ ). A radial distribution function (RDF) of the NP positions from TEM images gives maxima at 15.5, 25.2, and $48.8 \mathrm{~nm}$ (Figure S3, Supporting Information). The first peak corresponds to NPs in near contact, while the second and third correspond to an interlamellar spacing (d-spacing between two adjacent lamellae) of $10 \mathrm{~nm}$ indicating the crystallized PEO is compacted. However, there are potentially other explanations for these observations. First, drying artifacts are well known in TEM and the evaporation of trapped ethanol could potentially explain the spacing. ${ }^{[29]}$ Moreover, thiolated ligands on gold NPs have been shown to possess a degree of mobility although over longer timescales than used here. ${ }^{[35]}$ Therefore, the PEO must be more mobile than the anchoring group, otherwise the observed near contact of two NPs would not be possible. In a 2D assembly, as shown in Figure 2, the excess crystallized polymer could well be in the $z$-axis as opposed to in the plane of the assembly explaining the spacing observed in TEM. To determine if indeed there is any long-range order of the assembled NPs in situ, small-angle X-ray scattering (SAXS) measurements were undertaken.

The scattering intensity in SAXS is proportional to (a) the particle form factor $P(q)$ describing the size and geometry of the Au NP and (b) the structure factor $S(q)$ describing spatial correlation between the particles: $I(q) \propto P(q) S(q) .{ }^{[36]}$ The form factor of well-dispersed PEOcoated gold NPs was determined through the addition of water to NPs dispersed in ethanol, and measurement of their scattering curve. Above a certain threshold of water, PEO does not crystallize or display UCST behavior due to the complete hydration of the monomers, ensuring well-dispersed NPs. The quotient of the scattering curve of the assembled NPs over the form factor estimates the structure factor, as shown in Figure 1e. A characteristic oscillatory form at low $q$ is indicative of long-range order, and a maximum in the structure factor at $q=0.34 \mathrm{~nm}^{-1}$ corresponds to a length scale of 18-19 nm. This length scale is similar to the first maxima from the RDF of the TEM images, providing evidence the TEM figures are representative and the local structure observed are not 

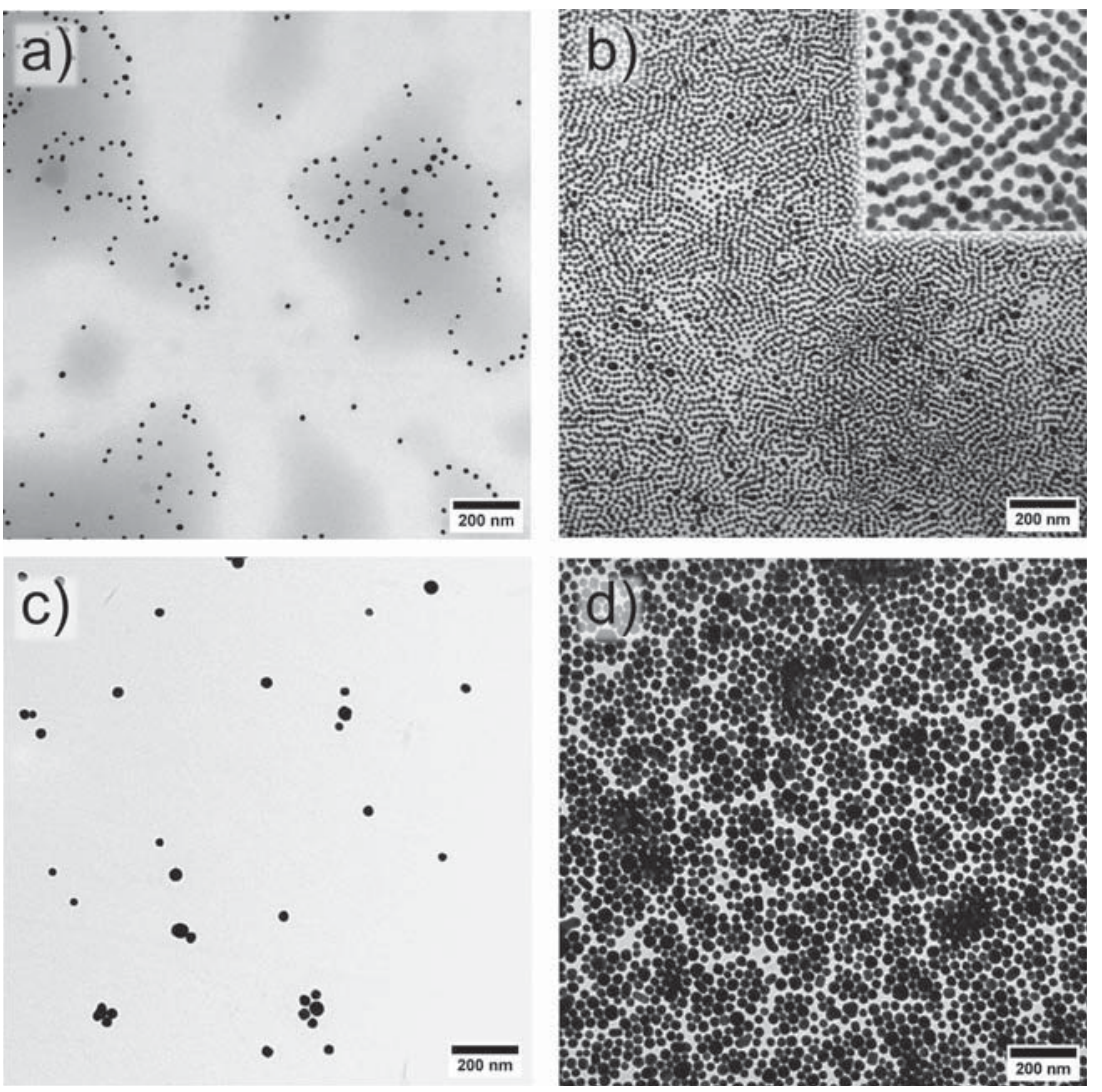

Figure 2. TEM of 16-nm-functionalized NPs dried either a) above or b) below the melting temperature of PEO in ethanol. Images were also taken of $33 \mathrm{~nm}$ NPs that were either c) dispersed or d) assembled.

artifacts. Due to the limited $q$ range of the instrument, it was not possible to probe longer length scales to investigate the more lamellar-like spacing.

The phase behavior of the polymer either dissolved in ethanol above or below the transition temperature was probed with wide-angle X-ray scattering (WAXS). As hypothesized, a crystal structure was detected with a peak at $q=13.57$ and $16.54 \mathrm{~nm}^{-1}$ corresponding to a d-spacing of 0.46 and $0.38 \mathrm{~nm}$, respectively (Figure 1f), consistent with the findings of other groups. ${ }^{[29,37,38]}$ Therefore, the polymer on the gold NP surface likely crystallizes into a well-ordered structure before reaching the UCST, at which point it would form an amorphous aggregate.

The self-assembly observed here differs from some other aggregating systems that have employed either LCST polymers, hydrogen bonding, or screening of electrostatic repulsion with salt to rely solely on van der Waals interactions for larger NPs. These systems characteristically arrest into large, nonergodic, aggregates without any long-range order. ${ }^{[2,39-44]}$ It is not immediately clear why this system presents more order with partially crystalline domains at the nanoscale. One reason may be the general guidelines that in order to undergo self-assembly: the interaction length scales should be large relative to the constituent particles, and the strength of the interaction cannot be too strong otherwise the particles are kinetically trapped and cannot reach an equilibrium structure. The longer range, and mobile, PEO crystallization would fit these guidelines while the shorter range van der Waals interactions are responsible for the near-contacting NPs observed in TEM. Using larger NPs with a similar PEO size will greatly increase the relative strength of the van der Waals forces, potentially resulting in a different structures forming. Indeed, for the case of the $33 \mathrm{~nm}$ gold NPs, there is less long-range order observed (Figure 2d), although local examples of hexagonal close packing could be found. We hypothesized the same would occur for smaller polymers, i.e., $2 \mathrm{kDa}$ with $16 \mathrm{~nm}$ gold NPs; however, a mixture of cubic and lamellar structures was observed indicating the curvature of the NPs may also control the structure. ${ }^{[4]}$

The melting hysteresis observed in both UV-vis-NIR spectroscopy and DLS was investigated as a function of PEO molecular weight and the concentration of water. An isothermal experiment was carried out at $20^{\circ} \mathrm{C}$ in ethanol with $1 \%$ water on either the dispersed or assembled NPs. The phase transition is rate independent over the time scales of interest, so that the assembled NPs stay assembled at $20{ }^{\circ} \mathrm{C}$ while the dispersed NPs stay dispersed (Figure S5, Supporting Information). Additionally, the crystallization transition can be shifted to lower temperatures by the addition of water, while the melting transition remains unchanged. The dependence of the crystallization transition on molecular weight of PEO is expected to originate solely from the different ratio of residual water to EO monomers present on each NP. To confirm this, we plotted the hysteresis curves as a function of the PEO to water ratio (Figure S6, Supporting Information).

The hysteresis observed is analogous to a shapememory polymeric switch. In this case, at intermediate temperatures the system is either purple and flocculated, or red and transparent depending on the thermal history. If the system has been heated up, even briefly, above the melting transition then the dispersion will be transparent and stable. However, a limitation of this is the easy resetting of the sensor if the temperature is once again dropped below the crystallization transition. An improvement to this system is the addition of calcium ions to the self-assembled NPs while they are below the 
a)
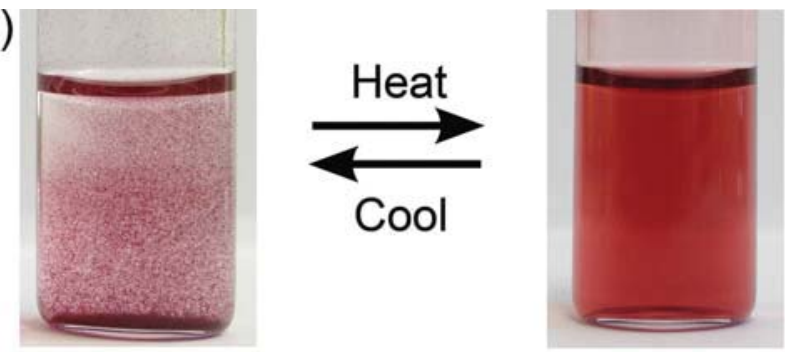

b)
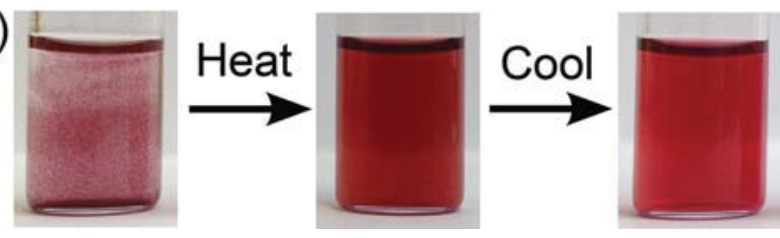

Figure 3. a) Thermoresponsive self-assembly of PEO-functionalized 16-nm NPs is fully reversible in absolute ethanol. b) Upon the addition of $\mathrm{CaCl}_{2}(0.2 \mathrm{M})$ to the preassembled NPs, the process is irreversible due to binding of $\mathrm{Ca}^{2+}$ to the PEO.

melting transition. Upon increasing the temperature, the NPs redisperse and the calcium ions bind to PEO, turning the neutral polymer into a cationic polyelectrolyte. Consequently, this charged PEO cannot crystallization and it becomes impossible to reset the system through cooling (Figure 3). Other cations, such as sodium, potassium, or magnesium (with the same counter ion), had no effect on the recrystallization of PEO on gold NPs.

In summary, we have demonstrated the self-assembly of PEO-functionalized gold NPs, by polymer crystallization, resulting in structures with long-range order. This is contrary to the typical self-assembled NPs functionalized with LCST or UCST polymers, which arrest into glassy or gel-like aggregates. The thermally reversible transition can be tuned by three parameters: water content, PEO molecular weight, and core NP size. This system has potential as a reversible thermally triggered smart material, made irreversible through the addition of calcium cations.

\section{Experimental Section}

Experimental materials and methods can be found in the Supporting Information.

\section{Supporting Information}

Supporting Information is available

Acknowledgements: This work was supported by the Swiss National Science Foundation (PP00P2_123373), the Adolphe
Merkle Institute, FriMat, and by the Swiss National Science Foundation through the National Centre of Competence in Research Bio-Inspired Materials.

Received: August 13, 2014; Revised: September 12, 2014; Published online: ; DOI: 10.1002/marc.201400457

Keywords: crystallization; gold; nanoparticles; reversible; self-assembly

[1] B. A. Grzybowski, C. E. Wilmer, J. Kim, K. P. Browne, K. J. M. Bishop, Soft Matter 2009, 5, 1110.

[2] K. J. M. Bishop, C. E. Wilmer, S. Soh, B. A. Grzybowski, Small 2009, 5, 1600.

[3] K. A. Dawson, Curr. Opin. Colloid Interface Sci. 2002, 7, 218.

[4] G. Foffi, G. McCullagh, A. Lawlor, E. Zaccarelli, K. Dawson, F. Sciortino, P. Tartaglia, D. Pini, G. Stell, Phys. Rev. E 2002, 65, 031407.

[5] E. C. Dreaden, A. M. Alkilany, X. Huang, C. J. Murphy, M. A. El-Sayed, Chem. Soc. Rev. 2012, 41, 2740.

[6] K. L. Kelly, E. Coronado, L. L. Zhao, G. C. Schatz, J. Phys. Chem. B 2003, 107, 668.

[7] Y. Wang, Y. F. Li, J. Wang, Y. Sang, C. Z. Huang, Chem. Commun. 2010, 46, 1332

[8] B. Pan, L. Ao, F. Gao, H. Tian, R. He, D. Cui, Nanotechnology 2005, 16, 1776.

[9] L. Wang, Y. Zhu, L. Xu, W. Chen, H. Kuang, L. Liu, A. Agarwal, C. $\mathrm{Xu}, \mathrm{N}$. A. Kotov, Angew. Chem. Int. Ed. 2010, 49, 5472.

[10] C. Raj, T. Okajima, T. Ohsaka, J. Electroanal. Chem. 2003, 543, 127.

[11] K. Nakayama, K. Tanabe, H. A. Atwater, Appl. Phys. Lett. 2008, 93, 121904.

[12] P. Cui, S. Seo, J. Lee, L. Wang, E. Lee, M. Min, H. Lee, ACS Nano 2011, 5, 6826.

[13] S. Shanmukh, L. Jones, J. Driskell, Y. Zhao, R. Dluhy, R. A. Tripp, Nano Lett. 2006, 6, 2630.

[14] J. Henzie, M. H. Lee, T. W. Odom, Nat. Nanotechnol. 2007, 2, 549.

[15] R. Sainidou, F. J. Garcia de Abajo, Opt. Express 2008, 16, 4499.

[16] T. K. Sau, C. J. Murphy, Langmuir 2005, 21, 2923.

[17] K. Park, H. Koerner, R. A. Vaia, Nano Lett. 2010, 10, 1433.

[18] Z. Sun, W. Ni, Z. Yang, X. Kou, L. Li, J. Wang, Small 2008, 4, 1287.

[19] K. Ai, Y. Liu, L. Lu, J. Am. Chem. Soc. 2009, 131, 9496.

[20] D. Nykypanchuk, M. M. Maye, D. van der Lelie, O. Gang, Nature 2008, 451, 549.

[21] M. E. Leunissen, C. G. Christova, A.-P. Hynninen, C. P. Royall, A. I. Campbell, A. Imhof, M. Dijkstra, R. van Roij, A. van Blaaderen, Nature 2005, 437, 235.

[22] M.-O. Zhu, L.-O. Wang, G. J. Exarhos, A. D. O. Li, J. Am. Chem. Soc. 2004, 126, 2656.

[23] J. Zhang, R. Pelton, Y. Deng, Langmuir 1995, 11, 2301.

[24] M. Beija, J.-D. Marty, M. Destarac, Chem. Commun. 2011, 47, 2826.

[25] R. R. Bhattacharjee, M. Chakraborty, T. K. Mandal, J. Phys. Chem. B 2006, 110, 6768.

[26] J. Seuring, S. Agarwal, ACS Macro Lett. 2013, 2, 597.

[27] E. E. Dormidontova, Macromolecules 2002, 35, 987.

[28] B.-J. de Gans, R. Kita, S. Wiegand, J. Luettmer-Strathmann, Phys. Rev. Lett. 2003, 91, 245501. 
[29] D. L. Ho, B. Hammouda, S. R. Kline, W.-R. Chen, J. Polym. Sci., B: Polym. Phys. 2006, 44, 557.

[30] C. Tabor, D. van Haute, M. A. El-Sayed, ACS Nano 2009, 3, 3670.

[31] C. Tabor, R. Murali, M. Mahmoud, M. A. El-Sayed, J. Phys. Chem. A 2009, 113, 1946.

[32] R. D. Groot, T. J. Madden, J. Chem. Phys. 1998, 108, 8713.

[33] M. Rubinstein, R. H. Colby, Polymer Physics, Oxford University Press, Oxford, 2010.

[34] J. U. Kim, M. W. Matsen, Macromolecules 2008, 41, 4435.

[35] P. Ionita, A. Volkov, G. Jeschke, V. Chechik, Anal. Chem. 2008, 80, 95.

[36] J. S. Pedersen, Adv. Colloid Interface Sci. 1997, 70, 171.

[37] C. Weiyu, K. Tashiro, M. Hanesaka, S. Takeda, H. Masunaga, S. Sasaki, M. Takata, J. Phys. Chem. B 2009, 113, 2338.
[38] R. S. Jagadish, B. Raj, P. Parameswara, R. Somashekar, J. Appl. Polym. Sci. 2013, 127, 1191.

[39] M. Y. Lin, H. M. Lindsay, D. A. Weitz, R. C. Ball, R. Klein, P. Meakin, Nature 1989, 339, 360.

[40] A. N. Shipway, M. Lahav, R. Gabai, I. Willner, Langmuir 2000, $16,8789$.

[41] D. S. Sidhaye, S. Kashyap, M. Sastry, S. Hotha, B. L. V. Prasad, Langmuir 2005, 21, 7979.

[42] K. L. Hamner, M. M. Maye, Langmuir 2013, 29, 15217.

[43] A. M. Mihut, J. J. Crassous, J. F. Dechézelles, S. Lages, A. Menzel, H. Dietsch, P. Schurtenberger, Polymer 2013, 54, 3874.

[44] C. Durand-Gasselin, M. Capelot, N. Sanson, N. Lequeux, Langmuir 2010, 26, 12321. 